\title{
Economic evaluation of patient navigation programs in colorectal cancer care, a systematic review
}

\author{
Chloé Gervès-Pinquié ${ }^{*}$ (D), Anne Girault ${ }^{2}$, Serena Phillips ${ }^{3}$, Sarah Raskin ${ }^{4}$ and Mandi Pratt-Chapman ${ }^{3}$
}

\begin{abstract}
Patient navigation has expanded as a promising approach to improve cancer care coordination and patient adherence. This paper addresses the need to identify the evidence on the economic impact of patient navigation in colorectal cancer, following the Health Economic Evaluation Publication Guidelines. Articles indexed in Medline, Cochrane, CINAHL, and Web of Science between January 2000 and March 2017 were analyzed. We conducted a systematic review of the literature using Preferred Reporting Items for Systematic Reviews and Meta-Analyses (PRISMA) guidelines. The quality assessment of the included studies was based on the Consolidated Health Economic Evaluation Reporting Standards (CHEERS) checklist. Inclusion criteria indicated that the paper's subject had to explicitly address patient navigation in colorectal cancer and the study had to be an economic evaluation. The search yielded 243 papers, 9 of which were finally included within this review. Seven out of the nine studies included met standards for high-quality based on CHEERS criteria. Eight concluded that patient navigation programs were unequivocally cost-effective for the health outcomes of interest. Six studies were cost-effectiveness analyses. All studies computed the direct costs of the program, which were defined a minima as the program costs. Eight of the reviewed studies adopted the healthcare system perspective. Direct medical costs were usually divided into outpatient and inpatient visits, tests, and diagnostics. Effectiveness outcomes were mainly assessed through screening adherence, quality of life and time to diagnostic resolution. Given these outcomes, more economic research is needed for patient navigation during cancer treatment and survivorship as well as for patient navigation for other cancer types so that decision makers better understand costs and benefits for heterogeneous patient navigation programs.
\end{abstract}

Keywords: Colorectal cancer, Patient navigation, Cost-benefit analysis, Health care costs

\section{Introduction}

The impacts of cancer on individuals, caregivers, society and health care systems are profound. The National Cancer Institute estimates that in 2016, 1.6 million people in the United States will be diagnosed with cancer and nearly 600,000 will die from the disease [1]. Globally, over eight million lives lost and almost 200 million disability-adjusted life years were attributed to cancer in 2013 [2]. Close to $\$ 125$ billion was spent on cancer care in the U.S. in 2010 [1], a figure anticipated to reach $\$ 173$ billion by 2020 [3].

\footnotetext{
*Correspondence: chloegerves@gmail.com

${ }^{1}$ Research Institute for Environmental and Occupational Health (Irset-Inserm UMR1085), Ester Team - UFR Santé - Département de Médecine, Rue Haute de Reculée, 49045 ANGERS Cedex01, France

Full list of author information is available at the end of the article
}

The growing cost of cancer care reflects successes in the field, such as increases in both the percentage of people who survive cancer and the number of years survived, with resultant costs of specialized care needs [4]. It also reflects failures: for example, inadequate coordination of care through an "increasingly specialized and fragmented health care system" [5], which can lead to service duplications, lower treatment adherence, poorer care quality, worse health outcomes, and increased costs for patients and payers [6-8].

Cancer cost must be considered in the context of health and health care disparities. Racial/ethnic minorities, low-income populations, and others from historically marginalized backgrounds tend to be diagnosed at later stages of disease progression, receive lower quality care and bear 
a disproportionate burden of disease. Racial/ethnic disparities in cancer cost an estimated annual \$193 billion in premature death and $\$ 471.5$ million in lost productivity in the United States alone [9]. As others have observed, there are both economic and moral arguments for bending the cost curve of cancer care [10]. Patient navigation (PN) has rapidly expanded as a promising approach to address cancer disparities, reduce the overall cost of cancer, and improve care coordination and patient adherence across the care continuum, particularly among minority and/or economically disadvantaged patient populations $[11,12]$.

PN programs have been effective in improving clinical outcomes and patient experience, including reducing patient distress and anxiety, shortening acute hospital stays, increasing patient satisfaction and empowerment, and reducing disparities in timely movement through the cancer care trajectory [13]. Yet PN's effects on the cost of cancer care are not as well documented. Few studies on PN programs provide an exhaustive economic evaluation of their outcomes, and even fewer base their evaluation on validated methodological guidelines like the Consolidated Health Economic Evaluation Reporting Standards (CHEERS) and on well-defined coordination problems [14]. More rigorous economic analyses of PN are needed for a variety of reasons, not least to inform policy decisions about if and how to pay for PN services, which in the U.S. are currently not reimbursed by third party payers.

Strengthening understanding of the economic impacts of PN is particularly valuable for cancer types in which population-level early detection is cost-effective and PN improves adherence to initial phases of care. Colorectal cancer (CRC) is the third most commonly diagnosed cancer in the United States and worldwide. CRC will be diagnosed in an estimated 96,000 people in the United States in 2017 and will take the lives of over 50,000 people, disproportionately affecting racial/ethnic minorities and economically disadvantaged people due to later-stage diagnosis and low screening adherence [15]. Globally, almost 694,000 lives were lost to CRC in 2012 [2]; it is estimated that worldwide CRC diagnoses will nearly double in the next two decades to reach 2.4 million cases in 2035. The United States will spend approximately $\$ 17.41$ billion on CRC care in 2020, with over half of cost spent on continuing care and in the last year of life [3]. Yet, the majority of spending on treatment, as well as the estimated $\$ 4.2$ billion in productivity lost to CRC deaths and inestimable individual and family suffering [16], is largely considered avoidable due to the success of screening and removal of pre-cancerous polyps.

PN has demonstrated improvements in timely movement through the CRC care trajectory, particularly among racial/ethnic minority, low-income, and other disadvantaged populations [17]. Accordingly, it makes an excellent case study to examine the economic impacts of $\mathrm{PN}$ on care.

Our study aimed to analyze the literature and assess the level of evidence on the economic evaluation of PN programs in CRC.

\section{Methods \\ Review process}

A systematic search of the scientific literature was conducted in four major databases (MEDLINE using PubMed, Web of Science, Cochrane and CINAHL) to identify relevant English-language publications relating to economic evaluations of PN programs in CRC. The Preferred Reporting Items for Systematic Review and Meta-Analysis (PRISMA) guidelines were used to ensure systematic selection of studies [18] (see Additional file 1: Table A).

The three preconditions for inclusion were that the study:

(1) evaluated PN services: we confirmed that each article explicitly addressed PN (including navigators with and without a clinical license such as nurses and social workers performing navigator functions) rather than other health care provider roles that may perform similar tasks,

(2) conducted an economic evaluation, and

(3) focused on CRC.

Keywords were defined according to population, intervention/comparator, outcomes, study design elements (see Additional file 1: Table B). Keywords were searched in the title or abstract of full-length publications that were published between January 2000 and march 2017.

Articles were excluded if they did not correspond to the above criteria. Systematic literature reviews were also excluded

\section{Study selection}

Our initial search resulted in 243 articles that met the above-mentioned criteria. The retrieved studies were reviewed by four researchers in close consultation with a senior author (MPC) and, in case of disagreement, issues were resolved by consensus.

Duplicates were removed, resulting in a total of 121 articles for review. The 121 citations were screened on the basis of titles and abstracts. 16 papers were then selected.

The full-text articles for the 16 abstracts selected for inclusion were retrieved and read. The final number of original empirical studies was 9 after assessment of eligibility for inclusion.

Data was extracted independently by four researchers. Extracted information included: bibliographic details, 
information on participants, PN interventions, outcomes, study design, and results. Disagreements were resolved by discussion.

Figure 1 provides a PRISMA diagram illustrating details of the search strategy.

\section{Quality assessment}

The studies identified for inclusion were assessed against the 24 key criteria contained in the CHEERS checklist [19]. The checklist has been jointly endorsed by ten journals. All items were presented in the tables for this review, consisting of five broad categories: Title and abstract (2 items); Introduction (1 item); Methods (14 items); Results (4 items) and Discussion (3 items) (see Additional file 1: Table C).

In certain studies, we considered that some CHEERS' items were not applicable:

- When the time horizon was less than one year, discounting (item 9) was considered not applicable

- When the economic evaluation was a cost analysis, effectiveness measurement (item 11) was considered not applicable

- When measured outcomes were not preferencebased, preference measurement (item 12) was considered not applicable.

We used the results of the quality assessment for descriptive purposes and to investigate potential sources of heterogeneity.

\section{Cost classification used}

The costs considered were:

- Direct costs encompass all the health care expenditures generated by the program. They include the resources used for program implementation (program cost) and both the

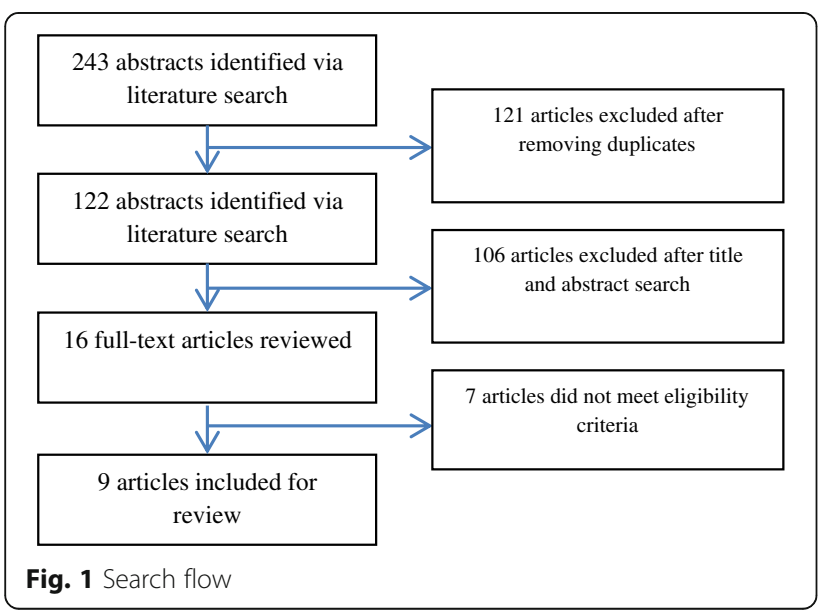

medical and non-medical resources generated as a consequence of the program (e.g., physician consultations, treatment cost, professional home care). These resources are priced on the health care market (consultation cost, treatment cost, etc.).

- Indirect costs correspond to resources without a market price, such as opportunity costs for both the patient (e.g., travel time, waiting time, and productivity loss on the labor market) and his/her relatives (since informal care time means the caregiver cannot pursue other activities). While necessarily estimated, these resources are given a monetary value to be integrated within the costs of the economic evaluation.

\section{Results}

We present in Additional file 1: Table $\mathrm{C}$ the quality assessment of the included studies based on the CHEERS checklist. It shows that seven out of the nine studies reviewed can be considered high quality studies, following an existing approach to determining quality in cancer scholarship [20], with an average proportion of $84.8 \%$ of checklist criteria fulfilled.

Table 1 shows the main characteristics of the studies included [21-29]. Most articles $(n=6)$ exclusively addressed navigating those due for recommended CRC screening to receive those services $(n=6)$. The few articles examining $\mathrm{PN}$ to diagnostic resolution $(n=2)$ addressed multiple cancer types. Two studies compared PN to screening colonoscopy versus other screening modalities (fecal occult blood testing (FOBT) or fecal immunochemical testing (FIT)). All studies but one occurred in the United States and took place in various clinical settings, primarily in the health care safety net setting. At least two-thirds of studies focused on racial/ethnic minority, low-income, or otherwise underserved populations. The only study to address PN from confirmed diagnosis through treatment or end of life occurred in New Zealand.

Navigator profiles and roles described in the articles were diverse. Three studies used nurse navigators; four used non-clinically licensed navigators with various titles such as "lay" health educator or outreach worker. One article included a licensed clinical social worker and at least two employed bilingual staff. For the seven studies that described navigator actions, navigators provided assistance through a wide range of tasks. These included identification and removal of barriers to care, coordination of appointments and referrals, appointment reminders, support and encouragement, information and education, and tracking and follow up. Among the reviewed studies, four were based on randomized controlled trials (RCTs). All the studies reviewed indicated the time horizon for evaluation. 


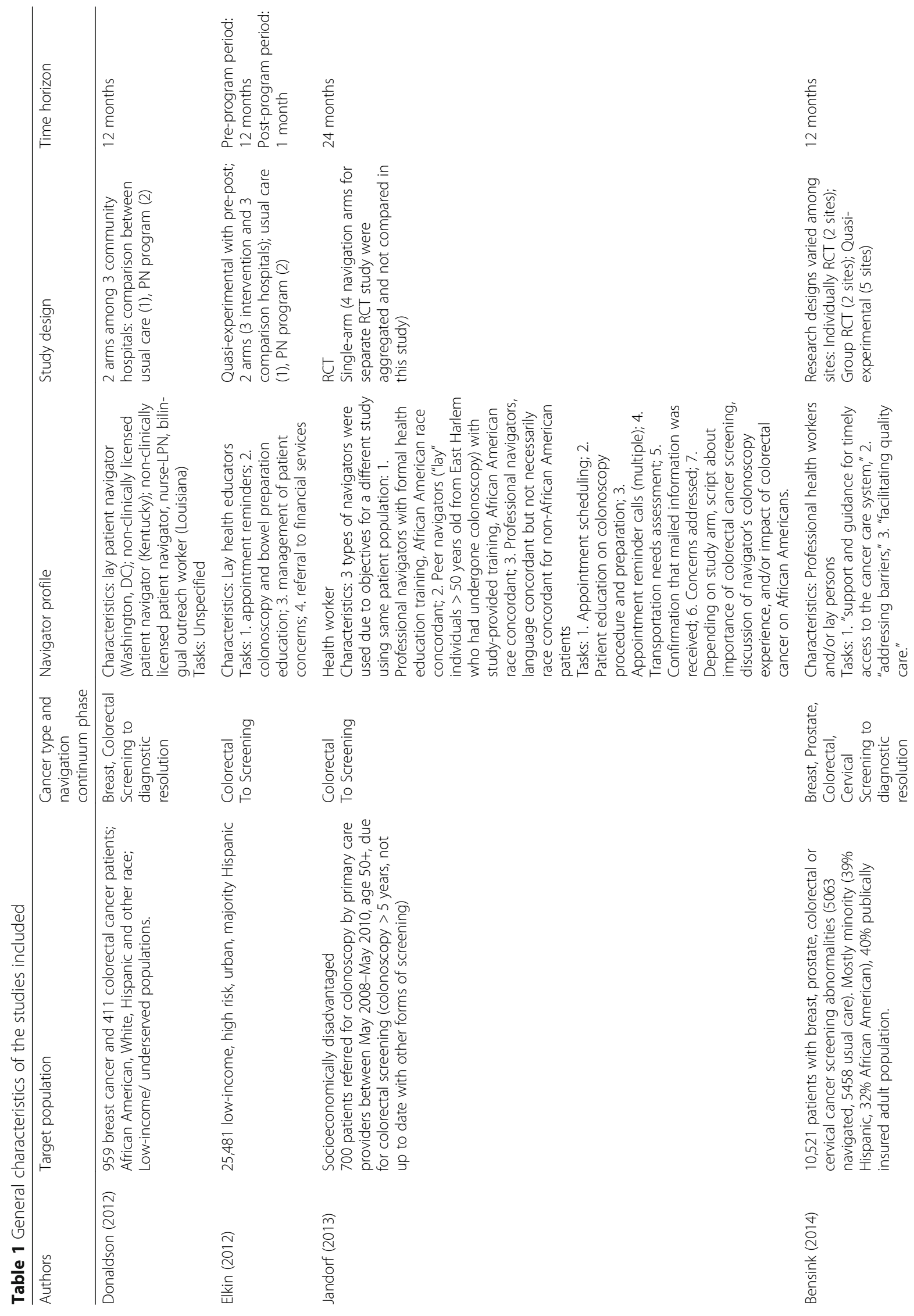




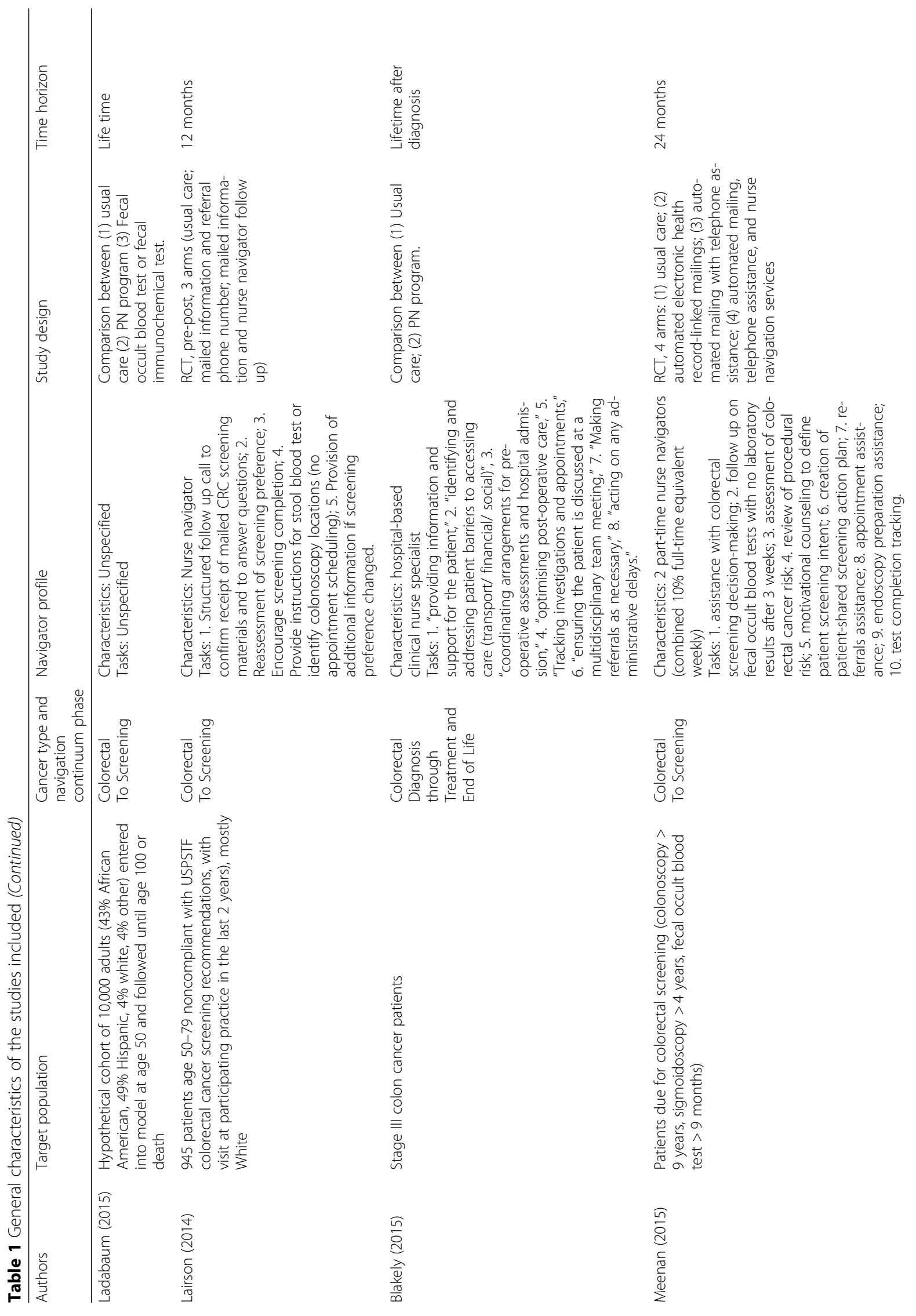




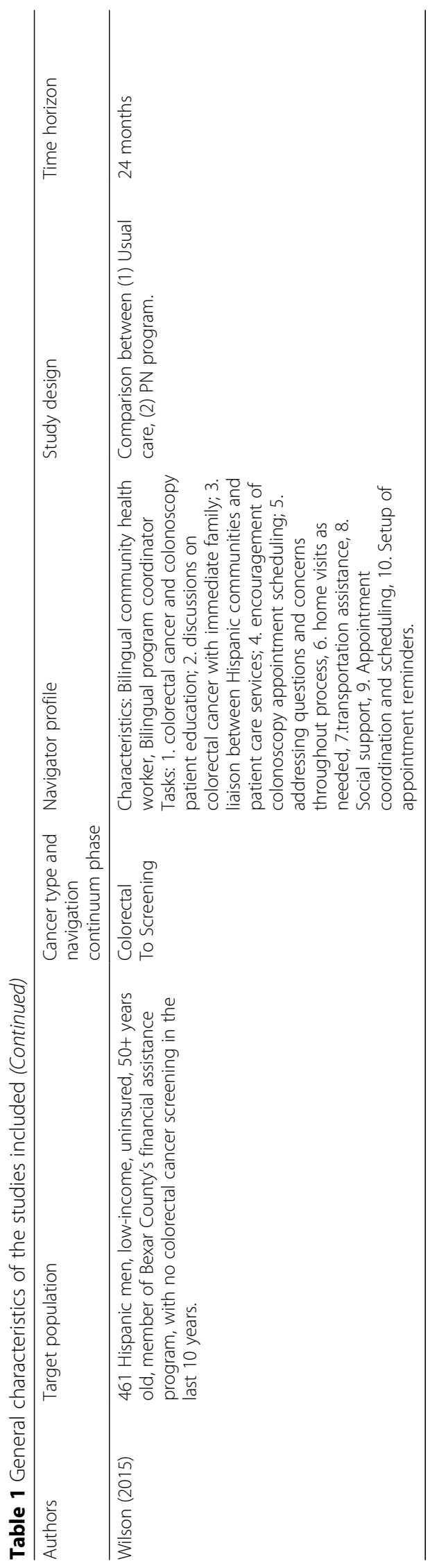


Table 2 shows that most of the studies $(n=8)$ adopted the health care system perspective, which refers to a variety of entities including the hospital $(n=3)$ or public or private payers $(n=5)$. Six studies were presented as cost-effectiveness analyses (among which, one presented both a cost-effectiveness analysis and a cost-benefit analysis), one was presented as a cost-utility analysis, one was a cost-consequence analysis, and one was a cost analysis. If we assume that using Quality Adjusted Life years (QALYs) implies conducting a cost-utility analysis [30], two of the cost-effectiveness analysis reviewed were also cost-utility analyses.

All studies computed the direct costs of the program, which were defined a minima as the program costs, including training, personnel, and supply costs. Eight studies considered direct medical costs, which were usually divided into outpatient and inpatient visits, tests and diagnostics. Estimated treatment cost was only considered in four papers and no study included direct non-medical cost, such as home care expenses. Only one study included indirect costs in the total costs associated with the PN program, including patient productivity loss and travel cost. No study included indirect costs associated with informal care. The clinical outcomes studied were mainly measures of time from abnormal finding to diagnostic resolution $(n=2)$, receipt of colonoscopy $(n=4)$, Quality Adjusted Life Years $(n=3)$ or Life years $(n=1)$. One third of the studies interpreted their results in relation to different stakeholders' willingness to pay (WTP) for improvements in care.

All but one study concluded that PN programs were unequivocally cost-effective for the health outcomes of interest. For instance, Incremental Cost Effectiveness Ratios (ICERs) ranged from $\$ 65$ to $\$ 1958$ per additional screening meaning that adopting the PN program instead of the alternative care strategy considered (for instance usual care, or fecal occult blood test or automated electronic health record-linked mailings) leads to a cost of $\$[65$ to 1958] for an additional screened patient. ICERs ranged from $\$ 1192$ to $\$ 9708$ per diagnostic resolution and from $\$ 3765$ to $\$ 15,600$ per QALY gained. There was high probability for PN to be cost-effective for CRC if stakeholder's WTP ranged between $\$ 1200$ and $\$ 1697$ per additional screening and from $\$ 16,500$ to $\$ 21,000$ per QALY gained. In comparison, the National Institute for Health and Clinical Excellence (NICE) has been using a cost-effectiveness threshold ranging between $£ 20,000$ and $£ 30,000(\$ 27,000-\$ 40,000)$ - usually per QALY gained - for over 14 years [31, 32]. The remaining study concluded that PN programs were only likely to be cost-effective (at $\$ 43,520$ per life-year saved) under the most favorable assumptions, in which patients lost to follow-up have more advanced cancer, and navigators account for a 6-month earlier time to diagnostic resolution and have a $15 \%$ higher probability of follow up resolution completion [24].

\section{Conclusions}

Most PN programs for CRC presented in our review had high probability for being cost-effective compared to usual care, given a conservative cost-effectiveness threshold of $\$ 50,000$ per QALY gained [33]; one study found one-time PN to be cost- saving. Cost-effectiveness evidence is most robust for PN programs designed to increase adherence with CRC screening using colonoscopy. Given the U.S. Preventive Services Task Force's A grade recommendation of CRC screening and the demonstrated success of PN to increase screening adherence among racial/ethnic minorities, low-income populations, and other disadvantaged patients, the volume and strength of the evidence in favor of the economic value of $\mathrm{PN}$ for colorectal screening adherence is unsurprising.

There are fewer articles for other phases of the continuum or using other screening methods [34]. The scant evidence seems to be tentatively favorable for the phase from abnormal screening to diagnostic resolution. Donaldson et al. (2012) concluded that PN programs increased achievement of timely diagnostic resolution for CRC (as well as breast cancers) among largely uninsured patients, and would be cost-saving if they were able to avert three to four cancer deaths per year [21]. Bensink et al. (2014) found limited economic benefit for PN during this phase (across four cancer types), indicating the greatest cost-effectiveness for those with the greatest needs such as the longest lapses in follow-up after screening, the most severe screening results, or the greatest potential to make gains in timeliness [24]. Lairson et al. advised payers to consider covering the costs of patient navigation for colonoscopy, which, compared to FOBT, has more chance to be considered cost-effective and even cost-saving when adopting larger time horizons [25]. The only study examining PN during the treatment phase addressed stage III colon cancer patients. Blakely et al. found PN to have high probability of cost-effectiveness, even considering a conservative WTP threshold [26]. These findings provide initial promising evidence for decision makers in support of PN for patients with more advanced cancers, and also for $\mathrm{PN}$ roles in providing treatment coordination and support.

Evidence of PN's cost-effectiveness is bolstered by the methodological soundness of the studies included in this review. Seven studies, all published after 2012, meet standards for high-quality based on CHEERS criteria. One of the studies was a cost analysis, making an incremental interpretation of the results impossible according to CHEERS guidelines [23].

Although there have been calls for establishing common PN cost measures [35], establishing such measures 


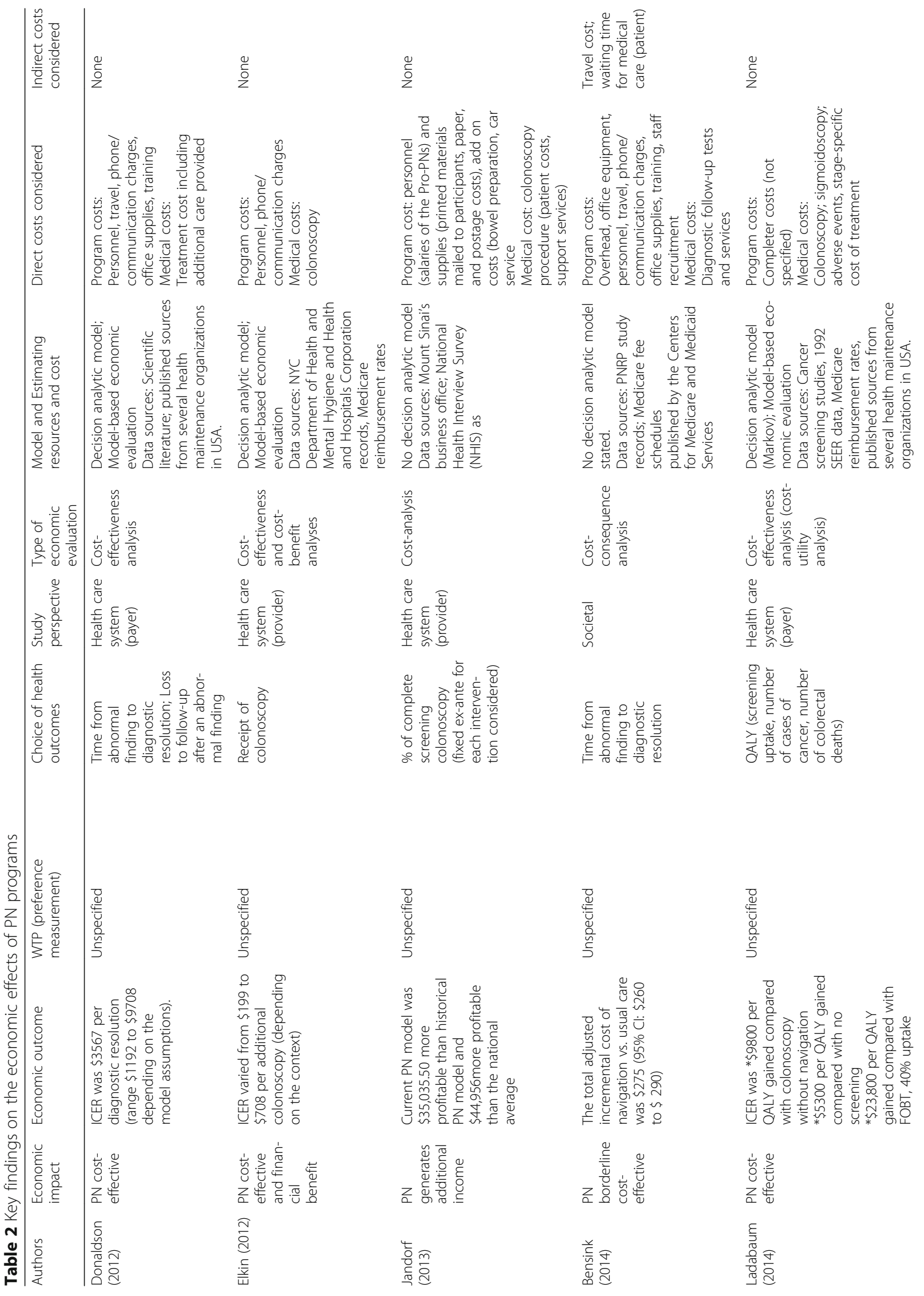




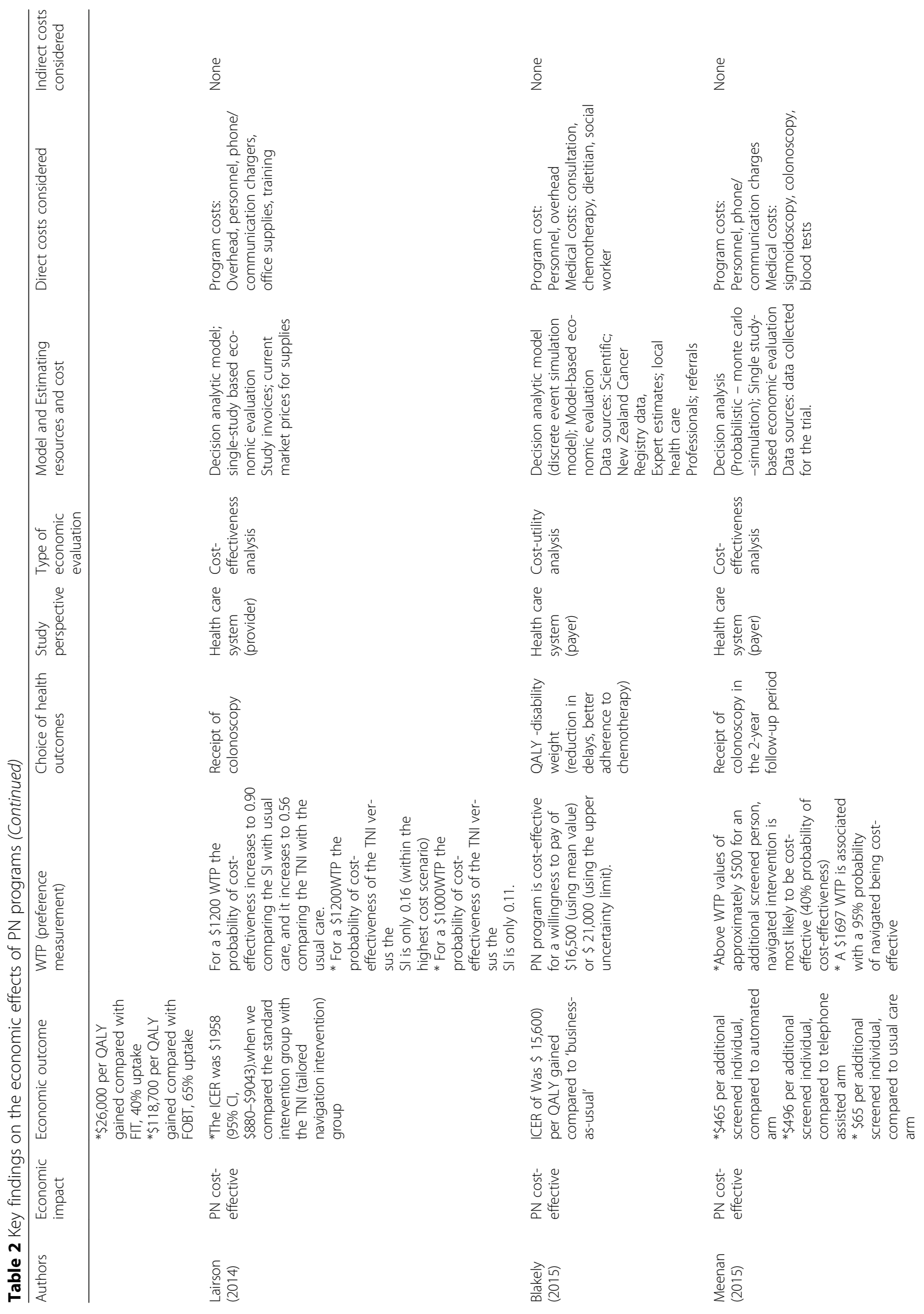




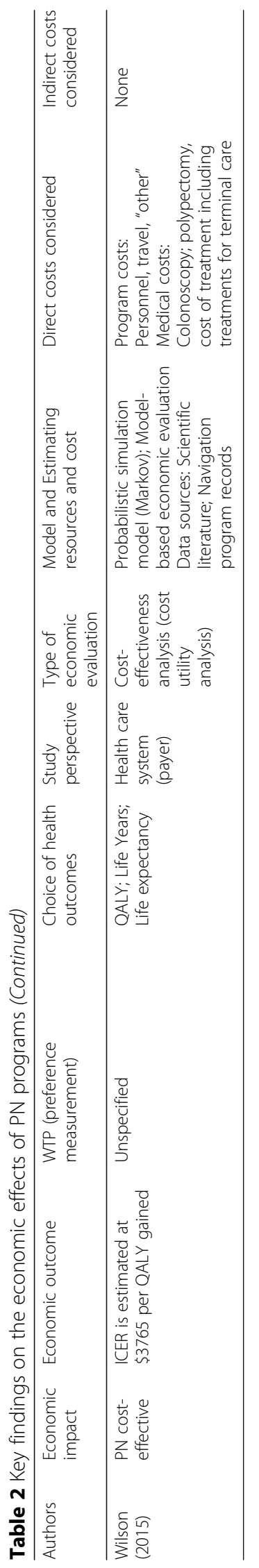


are challenging since costs to one stakeholder is revenue for another. In the studies we reviewed, there were variations in considerations and definitions of direct costs, indirect costs and health outcomes. The lion's share of the total cost of PN programs was most often attributable to direct medical costs rather than direct non-medical costs or indirect costs not covered by health insurance [36]. In other words, most of the reviewed studies adopted the health care system perspective rather than society's perspective.

Only a third of the articles addressed stakeholders' WTP. WTP is an important consideration to help payers optimize resource allocation, in particular with PN programs that are more costly but also more effective [28]. The perspective adopted is also crucial to a discussion of WTP thresholds, especially since PN programs considered to be cost-effective for society may exceed a hospital administrator's budget constraint, or their WTP, corresponding to their preferences for an improvement in patient's health outcome thanks to PN. It is noteworthy that patient preferences and patient reported outcomes (PROs) associated with PN are not addressed in the studies reviewed.

While this review advances understanding of the cost-effectiveness of CRC PN, findings should be interpreted with caution given limitations to current extant research. The heterogeneity of PN programs impedes the generalizability and comparability of individual and aggregate findings. The diversity of navigator roles, modes of communication and intensity of the interventions not only have the potential to produce heterogeneity in PN outcomes; it also produces variation in direct costs related to personnel and program costs. In settings in which PN occurs within a multi-faceted approach isolating $\mathrm{PN}$-specific outcomes from aggregate outcomes may be especially challenging [23, 35]. For instance, the intervention described by Wilson and Villarreal to increase colonoscopy adherence includes free colonoscopies, extended clinic hours, and taxi services [29]. Another problem affecting the generalizability of the results is the definition of usual care which was appreciably different across the studies reviewed. Further research could consist in comparing PN programs by navigator profile in addition to (or even instead of) being limited to a specific pathology. This kind of comparison would require detailed characteristics about navigator profile, such as their academic background, professional training, level of remuneration, length of work experience, etc. that are missing in most of the studies reviewed.

While PN program implementation is characterized by significant variability, the screening method and phases of the cancer continuum studied were limited among the studies examined. Therefore, these cost-effectiveness evaluation results may not apply for PN interventions with screening methods and at cancer continuum phases not included in this review. Extrapolation of findings for PN cost-effectiveness for other types of cancers should be done with extreme caution given that colonoscopy screening doubles as a preventive procedure, extending savings of early detection and removal of polyps over a lifetime. Colonoscopy is thus unique among cancer screening modalities.

Finally, our review faced several of the challenges often found in economic reviews. Economic modeling is complex. Multiple different models were used across the studies included in review, and results could have been affected by each model's type, structure, data sources and assumptions [37]. Lack of cost-benefit analyses prevented us from assessing whether PN could be profitable for providers, health care systems and societies (and at what cost for payers and possibly patients), but such analyses could move scholarship beyond cost-effectiveness.

\section{Additional file}

Additional file 1: Additional file of Economic evaluation of patient navigation programs in colorectal cancer care, a systematic review. (DOCX $38 \mathrm{~kb})$

\section{Abbreviations \\ CHEERS: Consolidated Health Economic Evaluation Reporting Standards; CRC: Colorectal cancer; FIT: fecal immunochemical testing; FOBT: fecal occult blood testing; ICER: Incremental Cost Effectiveness Ratio; PN: Patient navigation; PRISMA: Preferred Reporting Items for Systematic Review and Meta-Analysis; PRO: patient reported outcomes; QALY: Quality Adjusted Life years; RCT: randomized controlled trials; WTP: willingness to pay}

\section{Authors' contributions}

All authors were involved in all stages of literature search, study selection, and data analysis. First draft manuscript was written by CGP and AG. SP, SR, and MPC revised the manuscript critically for substantial intellectual content. All authors read and approved the final manuscript.

\section{Competing interests}

The authors declare that they have no competing interests.

\section{Publisher's Note}

Springer Nature remains neutral with regard to jurisdictional claims in published maps and institutional affiliations.

\section{Author details}

${ }^{1}$ Research Institute for Environmental and Occupational Health (Irset-Inserm UMR1085), Ester Team - UFR Santé - Département de Médecine, Rue Haute de Reculée, 49045 ANGERS Cedex01, France. ²EA MOS 7348 - French School of Public Health, 20 avenue George Sand, 93200 Saint Denis, France. ${ }^{3}$ Institute for Patient-Centered Initiatives and Health Equity at the George Washington University Cancer Center, 2600 Virginia Avenue NW, Suite 300, Washington, DC 20037, USA. ${ }^{4}$ L. Douglas Wilder School of Government and Public Affairs, Virginia Commonwealth University, Scherer Hall, \#313923 W. Franklin Street, Richmond, VA 23284, USA.

Received: 26 January 2018 Accepted: 7 June 2018 Published online: 14 June 2018

\section{References}

1. National Cancer Institute. Cancer statistics [Internet]. 2016. Available from: https://www.cancer.gov/about-cancer/understanding/statistics 
2. Fitzmaurice C, Dicker D, Pain A, Hamavid H, Moradi-Lakeh M, Maclntyre MF, et al. The global burden of Cancer 2013. JAMA Oncol. 2015;1(4):505.

3. Mariotto AB, Robin Yabroff K, Shao Y, Feuer EJ, Brown ML. Projections of the Cost of Cancer Care in the United States: 2010-2020. JNCl J Natl Cancer Inst. 2011;103(2):117-28. Available from: http://jnci.oxfordjournals.org/cgi/ doi/10.1093/jnci/djq495

4. Guy GP, Ekwueme DU, Yabroff KR, Dowling EC, Li C, Rodriguez JL, et al. Economic burden of cancer survivorship among adults in the United States. J Clin Oncol. 2013;31(30):3749-3757. Available from: http://www.ncbi.nlm. nih.gov/pubmed/24043731.

5. Wulff CN, Thygesen M, Søndergaard J, Vedsted P. Case management used to optimize cancer care pathways: a systematic review. BMC Health Serv Res. 2008;8:227. Available from: http://www.pubmedcentral.nih.gov/ articlerender.fcgi?artid $=2596122 \&$ tool $=$ pmcentrez\&rendertype $=$ abstract

6. Walsh J, Harrison JD, Young JM, Butow PN, Solomon MJ, Masya L. What are the current barriers to effective cancer care coordination? A qualitative study. BMC Health Serv Res. 2010;10:132. Available from: http://www. pubmedcentral.nih.gov/articlerender.fcgi?artid=2891740\&tool= pmcentrez\&rendertype $=$ abstract

7. Søndergaard EG, Grøne BH, Wulff CN, Larsen PV, Søndergaard J. A survey of cancer patients' unmet information and coordination needs in handovers-a cross-sectional study. BMC Res Notes. 2013;6:378. Available from: http:// www.pubmedcentral.nih.gov/articlerender.fcgi?artid=3850508\&tool= pmcentrez\&rendertype $=$ abstract

8. Kripalani S, Lefevre F, Phillips CO, Williams MV, Baker DW. Deficits in Communication and Information Transfer Between Hospital-Based and Primary Care Physicians. 2014;297(8):831-41.

9. Alexander B, Gurian G, Tilghman-Bryant T, Gaskin DJ, LaVeist TA, Richard P, et al. The societal and economic impact of cancer health disparities. Washington D.C.; 2014

10. Zafar YS. Financial toxicity of Cancer care: It's time to intervene. J Natl Cancer Inst. 2016 May;108(5) djv370

11. Natale-Pereira A, Enard KR, Nevarez L, Jones LA. The role of patient navigators in eliminating health disparities. Cancer [Internet]. 2011;117(15 Suppl):3543-3552. Available from: http://www.ncbi.nlm.nih.gov/pubmed/ 21780089

12. Percac-Lima S, Ashburner JM, Bond B, Oo SA, Atlas SJ. Decreasing disparities in breast cancer screening in refugee women using culturally tailored patient navigation. J Gen Intern Med [Internet]. 2013 Nov;28(11):1463-1468. Available from: http://www.ncbinlm.nih.gov/pubmed/23686510

13. Rodday AM, Parsons SK, Snyder F, Simon MA, Llanos AAM, Warren-Mears V, et al. Impact of patient navigation in eliminating economic disparities in cancer care. Cancer. John Wiley and Sons Inc. 2015;121(22):4025-34.

14. Husereau D, Jacobs P, Manns B, Hoomans T, Marshall D, Tamblyn R, et al. Economic evaluation of complex health system interventions: a discussion paper. 2014:38.

15. Siegel RL, Miller KD, Fedewa SA, Ahnen DJ, Meester RGS, Barzi A, et al. Colorectal Cancer Statistics, 2017. 2017;67(3):177-193.

16. Weir HK, Li C, Henley J, Joseph D. Abstract A89: Estimating potential years of life lost and productivity lost due to avoidable premature colorectal cancer deaths in U.S. counties with lower educational attainment. Cancer Epidemiol Biomark Prev. 2016;25(3 Supplement) A89-A89. Available from: http://cebp.aacrjournals.org/lookup/doi/10.1158/1538-7755.DISP15-A89

17. Domingo J-LB, Braun KL. Characteristics of effective colorectal Cancer screening navigation programs in federally qualified health centers: a systematic review. J Health Care Poor Underserved. 2017;28(1):108-26. Available from: https://muse.jhu.edu/article/648751

18. Moher D, Liberati A, Tetzlaff J, Altman DG, Grp P. Preferred reporting items for systematic reviews and meta-analyses: the PRISMA statement (reprinted from annals of internal medicine). Phys Ther. 2009;89(9):873-80.

19. Husereau D, Drummond M, Petrou S, Carswell C, Moher D, Greenberg D, et al. Consolidated Health Economic Evaluation Reporting Standards (CHEERS) statement. Eur J Heal Econ Elsevier.; 2013;14(3):367-372. Available from: https://doi.org/10.1016/j.jval.2013.02.010

20. Meregaglia M, Cairns J. Economic evaluations of follow-up strategies for cancer survivors: a systematic review and quality appraisal of the literature. Expert Rev Pharmacoecon Outcomes Res. 2015;7167:1-17. Available from: http://www.tandfonline.com/doi/full/10.1586/14737167.2015.1087316

21. Donaldson EA, Holtgrave DR, Duffin RA, Feltner F, Funderburk W, Freeman HP. Patient navigation for breast and colorectal cancer in 3 community hospital settings: an economic evaluation. Cancer. 2012;118:4851-9.
22. Elkin EB, Shapiro E, Snow JG, Zauber AG, Krauskopf MS. The economic impact of a patient navigator program to increase screening colonoscopy. Cancer. 2012;118(23):5982-8. Available from: http://www.ncbinlm.nih.gov/ pubmed/22605672.

23. Jandorf L, Stossel LM, Cooperman JL, Graff Zivin J, Ladabaum U, Hall D, et al. Cost analysis of a patient navigation system to increase screening colonoscopy adherence among urban minorities. Cancer [Internet]. 2013; 119(3):612-620. Available from: http://www.pubmedcentral.nih.gov/ articlerender.fcgi?artid=3492525\&tool=pmcentrez\&rendertype $=$ abstract.

24. Bensink ME, Ramsey SD, Battaglia T, Fiscella K, Hurd TC, McKoy JM, et al. Costs and outcomes evaluation of patient navigation after abnormal cancer screening: evidence from the Patient Navigation Research Program. Cancer. 2014;120(4):570-8. Available from: http://www.pubmedcentral.nih.gov/ articlerender.fcgi?artid=3946403\&tool=pmcentrez\&rendertype $=$ abstract.

25. Lairson DR, Dicarlo M, Deshmuk AA, Fagan HB, Sifri R, Katurakes N, et al. Cost-effectiveness of a standard intervention versus a navigated intervention on colorectal cancer screening use in primary care. Cancer. 2014;120(7):1042-9. Available from: http://www.ncbi.nlm.nih.gov/pubmed/ 24435411.

26. Blakely T, Collinson L, Kvizhinadze G, Nair N, Foster R, Dennett E, et al. Cancer care coordinators in stage III colon cancer: a cost-utility analysis. BMC Heal Serv Res. 2015;15:306.

27. Ladabaum U, Mannalithara A, Jandorf L, Itzkowitz SH. Cost-effectiveness of patient navigation to increase adherence with screening colonoscopy among minority individuals. Cancer. 2014;2015 Available from: http://www. ncbi.nlm.nih.gov/pubmed/25492455.

28. Meenan RT, Anderson ML, Chubak J, Vernon SW, Fuller S, Wang CYC-Y, et al. An economic evaluation of colorectal cancer screening in primary care practice. Am J Prev Med; 2015;48(6):714-721. Available from: https://doi.org/ 10.1016/j.amepre.2014.12.016

29. Wilson FA, Villarreal R, Stimpson JP, Pagán JA. Cost-Effectiveness analysis of a colonoscopy screening navigator program designed for Hispanic men. J Cancer Educ. Department of Health Services Research and Administration, College of Public Health, University of Nebraska Medical Center, Omaha, NE, United States; 2015;30(2):260-7.

30. Drummond MF, Sculpher MJ, Karl Claxton G, Stoddart L, Torrance GW. Methods for the economic evaluation of health care programmes, fourth edition. By. [internet]. Oxford: ox. Oxford University Press. 2015. Available from: https://global.oup.com/academic/product/methods-for-the-economicevaluation-of-health-care-programmes-9780199665877?q=Methods\%20for\% 20the\%20economic\%20evaluation\%20of\%20health\%20care\%20programmes \&lang $=e n \& c c=f r$.

31. McCabe C, Claxton K, Culyer AJ. The NICE cost-effectiveness threshold: what it is and what that means. Pharmacoeconomics [Internet]. 2008;26(9):733744. Available from: http://www.ncbi.nlm.nih.gov/pubmed/18767894.

32. Claxton K, Martin S, Soares M, Rice N, Spackman E, Hinde S, et al. Methods for the estimation of the National Institute for health and care excellence cost-effectiveness threshold. Health Technol Assess (Rockv). 2015:19(14):1504. Available from: https://www.journalslibrary.nihr.ac.uk/hta/hta19140/

33. Neumann PJ, Cohen JT, Weinstein MC. Updating Cost-Effectiveness - The Curious Resilience of the \$50,000-per-QALY Threshold N Engl J Med [Internet]. 2014;371(9):796-797. Available from: http://www.nejm.org/doi/ abs/10.1056/NEJMp1405158k

34. Jeong KE, Cairns JA. Review of economic evidence in the prevention and early detection of colorectal cancer. Heal Econ Rev. 2013;3:20.

35. Whitley E, Valverde P, Wells K, Williams L, Teschner T, Shih YC. Establishing common cost measures to evaluate the economic value of patient navigation programs. Cancer USA. 2011;117(15 Suppl):3616-23.

36. Subramanian S, Tangka FKL, Hoover S, Royalty J, DeGroff A, Joseph D. Costs of colorectal cancer screening provision in CDC's colorectal Cancer control program: comparisons of colonoscopy and FOBT/FIT based screening. Eval program Plann; 2017;62:73-80. Available from: https://doi.org/10.1016/j. evalprogplan.2017.02.007

37. Pignone M, Saha S, Hoerger T, Lohr KN, Teutsch S, Mandelblatt J. Challenges in systematic reviews of economic analyses. Ann Intern Med. 2005;142(12): 1073-80. 